\title{
On the Influence of Discrete Adhesive Patterns for Cell Shape and Motility: A Computational Approach
}

\author{
C. Franco $^{1}$, T. Tzvetkova-Chevolleau ${ }^{2}$ and A. Stéphanou ${ }^{1,2 *}$ \\ ${ }^{1}$ Laboratoire TIMC-IMAG, Equipe DynaCell, UMR CNRS 5525, Institut d'Ingénierie et de \\ l'Information de Santé (In3S), Pavillon Taillefer, Faculté de Médecine de Grenoble, 38706 La \\ Tronche Cedex, France \\ ${ }^{2}$ LTM, UMR CNRS 5129, c/o CEA Grenoble, 17 rue des martyrs, 38054 Grenoble Cedex 9, \\ France
}

\begin{abstract}
In this paper, we propose a computational model to investigate the coupling between cell's adhesions and actin fibres and how this coupling affects cell shape and stability. To accomplish that, we take into account the successive stages of adhesion maturation from adhesion precursors to focal complexes and ultimately to focal adhesions, as well as the actin fibres evolution from growing filaments, to bundles and finally contractile stress fibres.

We use substrates with discrete patterns of adhesive patches, whose inter-patches distance can be modulated in order to control the location of the adhesions and the resulting fibres architecture. We then investigate the emergence of stable cell morphologies as a function of the inter-patches distance, for two different cell phenotypes generated from the model. Force generated by the stress fibres on the focal adhesions and specifically the influence of the cell contractility are also investigated.

Our results suggest that adhesion lifetime and fibre growing rate are the key parameters in the emergence of stable cell morphologies and the limiting factors for the magnitude of the mean tension force from the fibres on the focal adhesions.
\end{abstract}

Key words: motility, stress fibres, focal adhesions, micropatterned substrates, integrative modelling.

AMS subject classification: 92B08, 92C17

\footnotetext{
*Corresponding author. E-mail: Angelique.Stephanou@imag.fr
} 


\section{List of abbreviations}

- AP: Adhesion Precursor;

- CB: Circular Bundle;

- DF: Dorsal Fibre;

- DSF: Dorsal Stress Fibre;

- ECM: Extracellular Matrix;

- FA: Focal Adhesion;

- FX: Focal complex;

- TA: Transverse Arc;

- TF: Transverse Fibre;

- TSF: Transverse Stress Fibre.

\section{Introduction}

The control of cell motility is one of the great challenges of cell biology since migration in its many forms (e.g. chematoxis or durotaxis) is crucial to many physiological and pathological events. However migration is just one aspect of motility. Indeed this term refers more generally to the ability for the cell to move spontaneously, that is to make movement by itself. Those movements do not necessary lead to migration. There are many circumstances in vivo where the non-migratory cell movements are as essential. For example the cell polarization and alignment in muscles or the cell membrane movements to maintain homeostasis in a tissue.

The in vitro study of cell membrane deformations and cell shape is essential to understand how the cell regulates its cytoskeletal architecture and the resulting forces to migrate. Intense research in the field has revealed the importance of the cell environment $[11,8,34,26]$. Recent breakthroughs in the micro/nanotechnology techniques, including the engineering of specific biomimetic matrices and substrates; in molecular biology, with the continuing discovery of new regulatory and transduction pathways, as well as in microscopy, with the development of new dynamic imaging techniques (FRET, FRAP, etc.), have on one hand accelerated knowledge acquisition on the mechanisms involved. In the other hand, these advances have further highlighted the complexity and highly integrated nature of cell motility. The up-to-date knowledge points out three essential and interrelated factors for motility: cell adhesion, cell cytoskeleton and cell environment.

Cell adhesions are the foci of cellular attachment [20]. They constitute the physical links between the extracellular matrix (ECM) and the actin cytoskeleton of the cell. They act as signalling 
hubs by conveying both biochemical and mechanical signals between the cell interior and the ECM. The actin cytoskeleton is a highly dynamic structure of filaments and fibres which can assemble and disassemble in response to these signals. The cytoskeleton contractile properties, resulting from the association of actin and myosin, allow the cell to generate tension forces and transmit them to the ECM via the adhesion sites. This gives the ability for the cell to sense the nature and mechanical properties of its surroundings.

The application of the micro and nano technologies in biological experimentation have broadened our understanding on the adhesion-actin cytoskeleton interactions by providing a powerful means to control the extracellular environment [11], either chemically (e.g. substrate functionalization with different ECM proteins), mechanically (e.g. substrate or matrix rigidity manipulation) [26], geometrically (e.g. shape and pitch length of adhesive pattern) [13] or topographically (3D adhesive structures with pillars or lines) $[33,35]$.

In parallel, mathematical models have proven to be useful tools for decoding cell motility processes and many have been proposed over the last few years. Mathematical models first aim at capturing cell motility features and subsequently at predicting cell behaviour or its dependence on specific key parameters. Mathematical models also allow to screen, through numerical simulations, a wide range of hypotheses thus sparing cost and time dedicated to laboratory experimentation. Nonetheless, the mere complexity of cell motility as a collective process has forced mathematical modelling studies to isolate and address only one or very few parameters at a time. For instance, many physical models describe the architecture [19, 21] and orientation [29, 17] of a static cell structure, such as the actin stress fibres network. Deshpande et al. [5, 6, 23] went further by proposing a bio-chemo-mechanical model for cell contractility, coupling stress fibre and adhesion turnover. However whole-cell models are still rare and lack integration of all concurrent intra and extracellular coupling events involved in motility. For instance, Kuusela and Alt (2009) take into account the actin and adhesion dynamics but neglect the maturation process of stress fibres [18]. Conversely, Coskun et al. (2007) consider the stress fibre dynamics to the detriment of adhesion dynamics [4]. The model proposed by Stéphanou et al. (2008) [32] and further extended in [30], which provides the basis for this paper, includes adhesion dynamics but neglects the explicit coupling of actin cytoskeleton to adhesions.

In this paper, we propose to improve the above biomechanical model of cell motility [30], by coupling the cell adhesion dynamics to the actin cytoskeleton remodelling. This time, the formation and maturation processes of actin fibres from the initial filament growth and bundling to the resulting mature stress fibres is explicitly taken into account. We note that our model only applies to describe cell motility on 2D substrates since the formation of mature focal adhesions and stress fibres rarely occur in 3D matrices [9]. In order to investigate the coupling between adhesions and actin fibres maturation processes, a discretized extracellular environment (i.e. substrate) with adhesive patches is considered. The substrate is rendered adhesive only at specific sites under the form of patches with predetermined geometry, size and spatial organization. A regular hexagonal network of small square patches is considered. The size of the patches $\left(4 \times 4 \mu m^{2}\right)$ is chosen so 
as to allow adhesions to cluster and grow while forming isolated adhesive islands [13]. The size of these islands is big enough to promote the formation of stress fibres. A range of inter-patches distance for the network are considered from 10 to $24 \mu \mathrm{m}$ so as to modulate the lengths of the fibre architecture. This parameter is essential to decipher force generation in the cell.

In the next section of this paper (section 2), we will present the biological background. We focus on the current knowledge on adhesions and fibres maturation and turnover and describe the key elements and events that serve as the basis to built our computational model. The model hypotheses and its mathematical description are described in section 3. Section 4 presents the simulation results and investigates specifically the importance of the inter-patches distance for cell shape, motility and force generation. Section 5 comments on the main results of this work and presents further possible extensions to elucidate the many remaining questions that need to be addressed to understand the full complexity of cell motility.

\section{Biological background}

Cell motility relies on the interactions between three main components: the actin cytoskeleton whose fibres organisation depends on the formation and distribution of the adhesions, which are themselves strongly dependent upon the extracellular environment and more specifically on the extracellular matrix mechanical and chemical properties (Fig. 1).

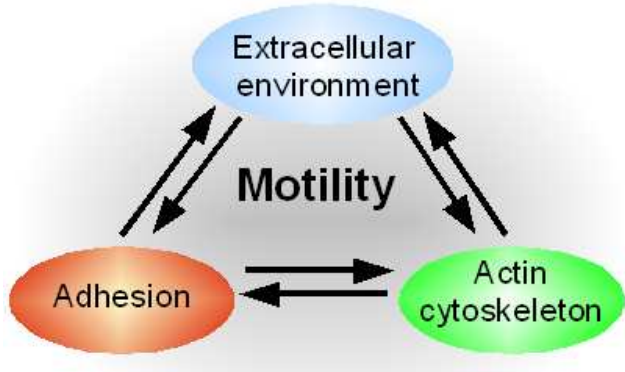

Figure 1: Adhesion, actin cytoskeleton and extracellular environment are three interacting elements controlling cell motility

To properly describe cell motility, it is therefore necessary to account for all three components and to investigate the tight coupling between them. In the subsequent sections we present the contribution of each of these three components from which our computational model is built.

\subsection{Formation and maturation of adhesions}

Adhesions are transmembrane proteins structures responsible for binding the cell to its environment, including other cells and the extracellular matrix. More than simple physical attachments, adhesions act as signalling hubs that detect, coordinate and transmit signals so that the cell can 
adapt its behaviour to the cues of its surroundings. Consequently, abnormal cell behaviours are often related to defects in the signalling cascade. Since we are interested in the motility of isolated cells, we will only consider cell matrix adhesions in this paper.

Adhesions are highly dynamic structures evolving in size and shape. Nearly ninety distinct proteins have been identified to contribute to individual adhesions [41], some being specific to one adhesion type. They can then be classified depending on their molecular composition [39, 25, 20]. Adhesion precursors (AP) form spontaneously. They correspond to the binding of an integrin receptor to ECM proteins such as fibronectin or collagen. APs form preferentially at the cell periphery on glass substrates, that is at the lamellipodium. The lamellipodium is an actively moving cell area where the membrane spreads over the 2D substrate [28]. APs dissociate with the passing movements of the membrane or mature by recruiting new proteins such as talin and paxillin to form a more stable adhesion type called focal complex (FX), which is strong enough to anchor the membrane to the ECM. As FXs grow, other proteins such as $\alpha$-actinin, FAK, VASP, Arp2/3 and vinculin are further recruited. These signalling and binding proteins allow the adhesion to nucleate actin filaments [36]. Mechanical stimulation on these adhesions, under the form of an increased tension force from the contractile cytoskeleton [10], causes adhesion maturation into focal adhesions (FAs) through the recruitment of zyxin and tensin. However, loss of tension leads to disassembly of these proteins. Although FAs are stable enough to support high stress from the contractile actomyosin complex required for cell translocation, they can also act as anchors restraining or even preventing cell migration.

\subsection{Formation and maturation of stress fibres}

Stress fibres are contractile actomyosin bundles and are the last stage of the fibre maturation process. They play a key role in cell motility by regulating adhesion turnover and exerting cell mechanical forces on the substrate. The maturation process of the actin cytoskeleton fibres involves the association of actin filaments with a range of specific proteins, such as myosin II, which confers the contractile properties of the actomyosin complex, tropomyosin, which regulates the binding of myosin to actin filaments, and $\alpha$-actinin which is a cross-linker involved in the attachment of the actin cytoskeleton to the membrane [27].

Different types of actin bundles can be identified in the cell, depending on their location, orientation (radial or transverse), size and thickness, contractile properties, and attachment to the cell structures (other cytoskeleton fibres or adhesions). Four types of bundles have been identified and described so far (see [22] for a review):

- transverse arcs, formed at the leading edge of the cell by the assembly of short bundles of actin. The high density of a branched network of actin in the lamellipodium nucleated by the Arp $2 / 3$ complex and its rapid turnover involving many actin regulating proteins, generates numerous transverse arcs. Myosin II is essential for the actin arcs structures formation and stabilization in the spreading margins and it is incorporated as the arcs flow backwards through the lamellipodia towards the base of the lamella. Transverse arcs are not attached to 
the substrate. Nonetheless, they can connect to sustrate-related structure and transmit tension to the environment.

- circular bundles, formed by the accumulation of the transverse arcs as they reach the lamellipodia. This is occuring at the early stage of cell spreading, typically one hour after plating the cells [27]. The contractility of circular bundles regulates the formation of focal adhesions in the spreading process.

- dorsal stress fibres, resulting from the elongation of actin filaments at focal adhesions. $\alpha$ actinin is a cross-linker involved in the formation of bundles of actin filaments. A dorsal fibre is therefore attached to a focal adhesion at one end and the other end elongates mostly in the radial direction by rising towards the dorsal surface of the cell [24]. It can ultimately connect to a circular bundle [27]. Despite the term "stress" associated to their name, dorsal stress fibres are not contractile, however they convey contractility once connected to a contractile structure (circular bundle or other contractile fibres).

- ventral stress fibres, formed mainly by the adjoining of two dorsal stress fibres. Ventral stress fibres are consequently anchored at both ends by a focal adhesion [14]. These fibres are located at the ventral side of the cell, that is next to the adhesion surface in vitro.

Figure 2 presents a diagram which synthesizes the main maturation phases of adhesions and fibres, as they will be implemented in the computational model. We make several simplifications. First, we do not consider the ventral stress fibres since they result from the adjoining of dorsal stress fibres only that latter fibre type is explicitly considered. Second, we strictly reserve the name of dorsal stress fibres (DSF) to contractile fibres. Consequently, unattached, that is non-contractile fibres are then named dorsal fibres (DF). Finally since the model is bidimensional, we do not differentiate the ventral side from the dorsal side of the cell. We assume that the fibre dynamic is all occuring in the same plane.

\subsection{Cell environment}

The cell environment determines cell fate and function [11]. It has been shown that, stem cells placed on substrates of different stiffness differentiate into distinct cell lineages [8]. Recent advances in micro and nano fabrication techniques and surface chemistry allow to construct artificial cellular microenvironments with precisely controlled mechanical characteristics, topography, and geometry. Specifically, the ability to manipulate the localization and shape of adhesive areas has expanded our ability to study the mechanisms governing cell shape [1]. Furthermore, discrete micropatterned substrates constructed with regularly distributed adhesive pillars or patches and coated with different binding proteins (e.g. fibronectin, collagen, etc.) are closer to the in vivo context, in which a cell adheres to the extracellular matrix when it encounters a binding protein. Using substrates with discrete adhesion sites, Guillou et al. (2008) [13] experimentally revealed the complex nature of membrane protrusion. First, filopodia grow until they reach an adhesion patch. Then filopodia consolidate and actin dynamics trigger membrane protrusion at the tip of the filopodia in contact with an adhesion site. By manipulating the shape and size of adhesive patterns 


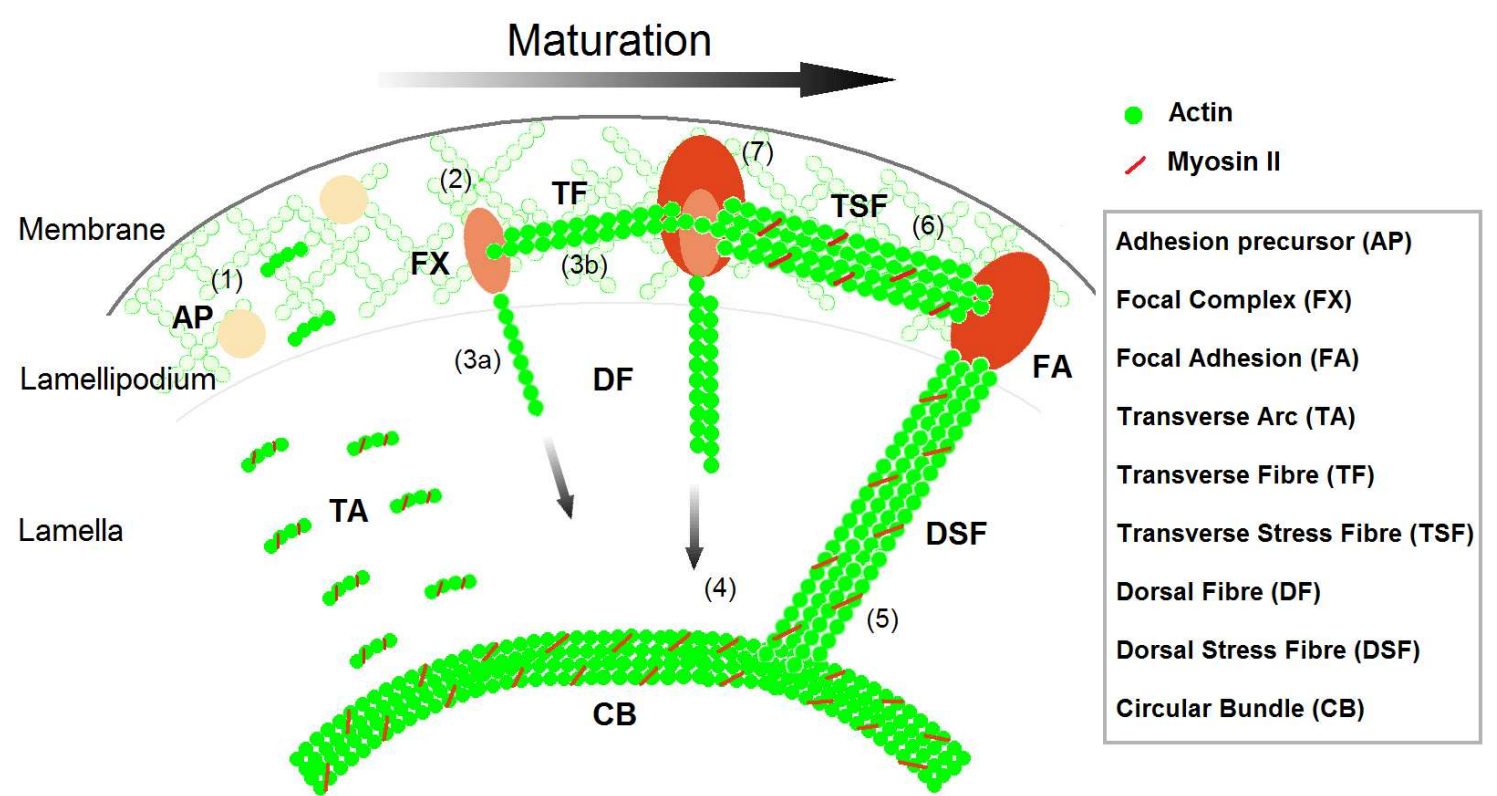

Figure 2: Seven different stages in the adhesion and actin fibre maturation can be clearly distinguished. Adhesion precursors (AP) form at the lamellipodium (1). In parallel, transverse arcs (TA) are formed from the shedding of the branched actin network. Then they flow towards the cell centre, become contractile by recruiting myosin II in the lamella and accumulate to form a circular contractile bundle (CB). An AP grows until it reaches a critical size and then matures into a focal complex (FX) (2). FXs are nucleation points of dorsal fibres (DF)(3a) and transverse fibres $(\mathrm{TF})(3 \mathrm{~b})$ in the radial and tangential directions respectively. A DF elongates until it connects to the CB (4). This connection makes it become contractile and mature into a dorsal stress fibre (DSF) (5). A TF linked to a FA recruits myosin II to become contractile and matures into a transverse stress fibre (TSF) (6). A TSF is linked at both sides by a FA (7).

(e.g., V-, U-, or T-shape), it is now known that stress fibres work against the membrane tension by forming above non-adhesive areas $[34,15]$. The clustering of FAs only at the substrate adhesive patches and their bridging with stress fibres influence cell polarization. Thus, cells geometry of actin cytoskeleton and consequently functional response, including migration, are directly depending upon adhesion site localization.

In order to decipher and understand how the maturation of cell adhesions is dependent upon the maturation of stress fibres and vice versa, we are considering a patterned extracellular environment formed by discrete adhesive patches, regularly arranged, in order to control the positioning and size of the adhesion zones of the cell which will in turn restrain the fibre lengths. By varying the inter-patches distance we are able to study how the environment is conditioning the stability of the cell shape and how this is related to cell force regulation, that is the magnitude of the force that the cell is able to develop and sustain. 
Our model for adhesion-fibre coupling is essentially based on results from the literature described in this section. However, we performed some preliminary experiments to assess the behaviour of fibroblast cells on the adhesive arrays we implemented and tested in our model. The aim of those experiments is to provide the qualitative baseline for our simulations. Murin 3T3 fibroblast cells are plated on a regular hexagonal network of adhesive patches with varying inter-patches distance (see Appendix A and B for details on cell culture and microfabrication of the adhesive patches respectively).

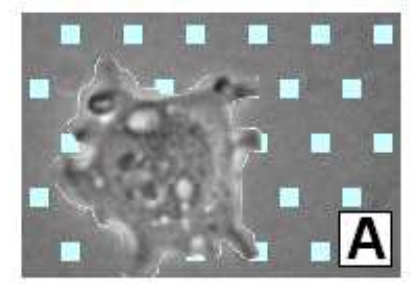

$\mathrm{t}=115 \mathrm{~min}$

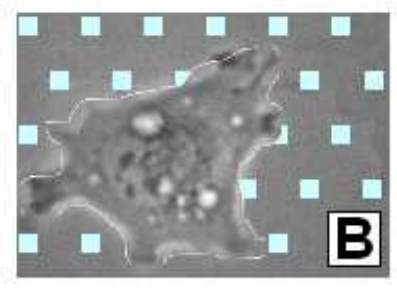

$\mathrm{t}=165 \mathrm{~min}$

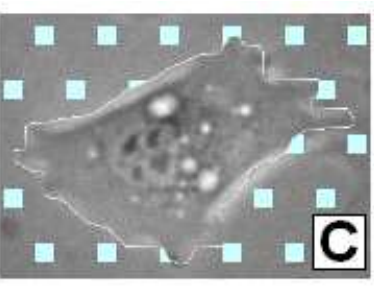

$\mathrm{t}=215 \mathrm{~min}$

Figure 3: Successive snapshots of a $3 \mathrm{~T} 3$ fibroblast cell plated on a network of adhesive patches, taken at time $t=115 \mathrm{~min}(\mathrm{~A}), t=165 \mathrm{~min}(\mathrm{~B})$ and $t=215 \mathrm{~min}(\mathrm{C})$. The inter-patches distance is $10 \mu \mathrm{m}$ and the patch size is $4 \times 4 \mu \mathrm{m}^{2}$. Adhesive patches are highlighted by graphical post-treatment with PhotoFiltre (c). The reference time $(t=0)$ corresponds to the instant of cell deposition on the substrate.

Figure 3 shows a typical example of the evolution of the cell shape on discrete adhesive patterns. The cell initially exhibits a round shape with several short protruding zones (A). The cell progressively spreads and forms new adhesions with more distant patches (B) which will ultimately constrain its shape to a more polarized and geometrical shape (C).

\section{Model}

Cell motility and the emergence of stable cell morphologies on discrete adhesive patterns are investigated with a computational model. The model is based on a set of partial differential equations to describe continuous cell membrane deformations resulting from the competition between protrusion and retraction forces, conditioned by actin turnover, with a cellular automaton. The latter integrates the inter-related maturation of focal adhesions and stress fibres which are by nature discrete components in the cell. This work thus further extends successive works by Stéphanou et al. (2004, 2008, 2009), where large membrane deformations [31], adhesion maturation [32] and the formation of actin stress fibres [30] were successively addressed.

\subsection{Cell membrane deformations}

The model considers the two-dimensional annular domain bounded by the cell nucleus on one side and the plasma membrane on the other side, with the latter defining a free-moving boundary. We 
denote by $L(\theta, t)$ the width of the annular domain along any radial direction $\theta(0 \leq \theta \leq 2 \pi)$ and time $t$ (Fig. 4), $a(\theta, t)$ is the free actin concentration at location $\theta$ and time $t$ in the lamellipodium and $v(\theta, t)$ represents its tangential velocity. Periodic boundary conditions apply to these variables.

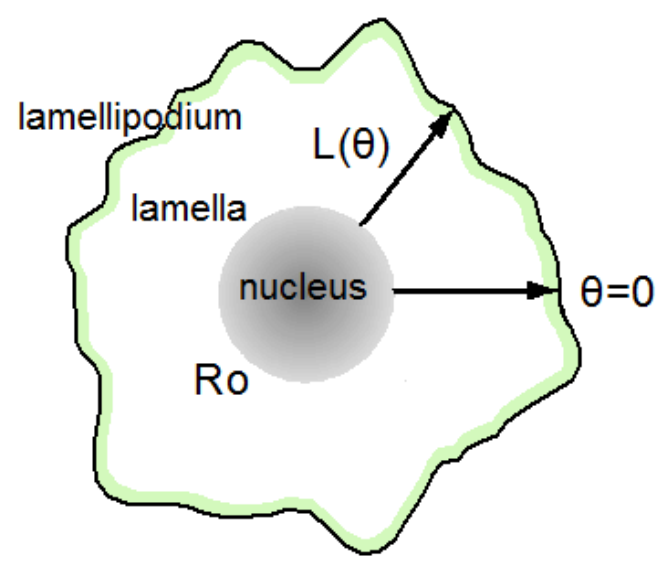

Figure 4: Theoretical representation of the cell. The cell nucleus (in grey) is represented by a circular domain with radius $R_{0}=7 \mu \mathrm{m}$. Two zones are distinguished $(i)$ the lamellipodium which is the narrow peripheral zone (green area) containing a dense network of interconnected actin filaments, and (ii) the lamella which is the zone located between the lamellipodium and the cell nucleus. Polar coordinates are used. The horizontal axis corresponds to $\theta=0 . L(\theta)$ is the distance between the nucleus and the membrane in any angular direction $\theta$.

The normalized system of partial differential equations ruling the movements of the cell membrane is given by:

$$
\begin{aligned}
\frac{\partial}{\partial t}(L a) & =-\frac{\partial}{\partial \theta}(L a v)+L(1-a) \\
a v & =\frac{\partial}{\partial \theta}\left[\mu a \frac{\partial v}{\partial \theta}+\sigma(a)\right] \\
\delta(a) \frac{\partial L}{\partial t} & =F_{\text {out }}+F_{\text {in }}+\kappa
\end{aligned}
$$

where the two first equations describe the actin turnover and its displacement in the lamellipodium, with $\mu$ the viscous coefficient for the cytoplasm and $\sigma(a)=\psi a^{2} \exp \left(-a / a_{\text {sat }}\right)$ the contractility of the actomyosin network, which locally depends on the actin density and whose magnitude is controlled by the contractility coefficient $\psi$. The third equation describes the movements of the cell membrane resulting from the force equilibrium, where $\delta(a), \kappa, F_{\text {out }}$ and $F_{\text {in }}$ represent the cell adhesive condition, curvature-induced tension of the membrane, the protrusion and the retraction forces respectively. These components are modelled on the basis of the following assumptions:

- the formation of an adhesion is translated by an increase friction between the cell membrane and the substrate, on top of the base friction assumed to be linearly depending upon the 
local actin density, that is $\delta(a)=a+\alpha \delta_{A P} . \alpha$ is the friction coefficient and $\delta_{A P}=0$ or 1 whether there is an AP, only adhesion type allowed to slide relatively to the substrate, or not. If the adhesion is a FX or FA then the membrane is tethered to the substrate which means $\partial L / \partial t=0$

- the cell membrane and the underlying actin network in the lamellipodium form a membraneactin cortex. This cortex can be considered as a reticulated structure modelled by a succession of springs between a discrete number of membrane points. The tension of each individual spring $i$ is given by $f_{i}=-k_{m}\left(l_{i}-l_{0}\right)$ with $k_{m}$ the elasticity coefficient of the membrane, $l_{i}$ the membrane length between two consecutive points and $l_{0}$ the resting length between points of the unstretched membrane (circular cell shape). The resulting curvature-induced tension influencing the cell membrane radial displacement are thus given by $\kappa=\hat{f}_{\text {left }}+\hat{f}_{\text {right }}$, where $\hat{f}_{\text {left }}$ and $\hat{f}_{\text {right }}$ are the radially projected contributions of the left and right springs at the considered membrane point, with $\hat{f}=f \cos \phi, \phi$ being the projection angle;

- the protrusion of the membrane is primary due to the cell hydrostatic pressure $\beta$. This term is reinforced by a repulsive force from the circular bundle, with intensity $\beta_{b}$, when the membrane approaches its vicinity, that is $L(\theta) \leq R_{\mathrm{CB}}+\varepsilon, R_{\mathrm{CB}}$ being the radius of the circle enclosing the $\mathrm{CB}$ and $\varepsilon$ the tolerance approach value. The resulting protrusive term is thus given by $F_{\text {out }}=\beta+\beta_{b} \delta_{\mathrm{CB}}(\theta)$ with $\delta_{\mathrm{CB}}(\theta)=0$ or 1 whether the vicinity condition of the membrane with the bundle is verified or not at membrane point $\theta$;

- the retraction is due to the actin network-membrane attachment and linearly depends upon the actin density, that is $-\gamma L a$, with $\gamma$ the elasticity coefficient for the actin network in the lamellipodium. Additionally if a TF is attached to the considered membrane point, it creates a tension force, similar to the membrane spring tension, modelled by $F_{\text {fibre }}=-k_{f}\left(l_{f}-\right.$ $\left.l_{f 0}\right)$, with $k_{f}$ the elasticity of the fibre, $l_{f}$ its length and $l_{f 0}$ the resting length of the fibre corresponding to its unstretched state. Once again, only the radial component of the force which contributes to cell membrane movements is considered, that is $\hat{F}_{\text {fibre }}=F_{\text {fibre }} \cos \phi$, with $\phi$ the radial projection angle. The resulting retraction force is then given by: $F_{\text {in }}=$ $-\gamma L a+\hat{F}_{\text {fibre }}$. If the TF ultimately matures into a TSF, we assume that the tension forces of the fibre underneath the membrane is strong enough at each of its ends so that the membrane spontaneously sticks to the newly created TSF.

\subsection{Formation and maturation of adhesions and fibres}

The formation, maturation and fate of adhesions and actin fibres are tigthly linked. That is why it is difficult to describe them independently. In this section we will describe how their dynamics (outlined in figure 2) is coupled to the model of membrane deformations and integrated into a computational framework (Fig. 5). We retain the same notation as in figure 2 to describe the seven maturation stages. 
Three adhesions types are differentiated in the model, depending on their maturation level, from AP to FA via FX. It is assumed that APs form spontaneously when the cell is in contact with an adhesive patch (Fig. 5.(1)). Thermal fluctuations can account for the energy required for the formation of this type of transcient and weak adhesion type. APs can easily break if the membrane moves too quickly or above a non-adhesive zone. We assume that AP matures into FX through a clustering process. The cluster grows until it reaches a critical size $S_{\text {crit }}$ above which it becomes a FX (Fig. 5.(2)).

FXs are stronger adhesions, which adhere the membrane to the substrate locally and therefore prevents membrane movements. Moreover, FX promotes the formation of a DF (Fig. 5.(3a)), that elongates in the radial direction (i.e. towards the cell centre) and the formation of a $\mathrm{TF}$ that is assumed to appear spontaneously between two consecutive and not too distant FXs (Fig. 5.(3b)), thanks to the readily available underlying actin network in the lamellipodium.

Finally, when mechanically solicited FX can mature into FA. We consider an implicit solicitation, occuring when the DF connect to the contractile CB (Fig. 5.(4)). In that case, traction force is conveyed by the DF from the $\mathrm{CB}$ to the parent adhesion FX which makes it mature into a FA, while the DF becomes a DSF (Fig. 5.(5)).

In parallel, when an adhesion matures into a FA, the TF connected to that FA spontaneously matures into a TSF (Fig. 5.(6)). TSFs are contractile and pull on the adhesions at both of their ends, thus allowing the FXs to mature into FAs (Fig. 5.(7)).

Stress fibres either DSF or TSF, are contractile and generate tension forces. If the resulting force $R_{\mathrm{F}}$ from these fibres measured at a FA is below a threshold tension then the FA disassembles triggering the simultaneous disassembly of all fibres connected to it.

\section{Limitations}

We note that our model exclusively considers peripheral adhesions since it is widely acknowledged that cell adhesions mainly form at the cell periphery, that is in the lamellipodium $[12,37]$ if we except podosomes and fibrillar adhesions [2]. Integrins located at a distance less than $0.5 \mu \mathrm{m}$ from the cell edge have been shown to have a higher affinity for the extracellular matrix. They consequently lead to more stable integrin-cytoskeleton links and are reinforced under force stimulation [16]. The formation of adhesions with patches located underneath the cell is not considered in the model. We thereby implicitly assume that they play a secondary role that remains to be investigated. We also note that a critical size criterion for integrin clusters is used in the computational model to mark the transition from AP to FX. However the model does not describe the physical growth of FX and FA. The growth of the adhesions allow them to progressively increase their resistance to traction that we take into account in a stepwise manner in the model without explicitly describing any changes in the adhesions size. 


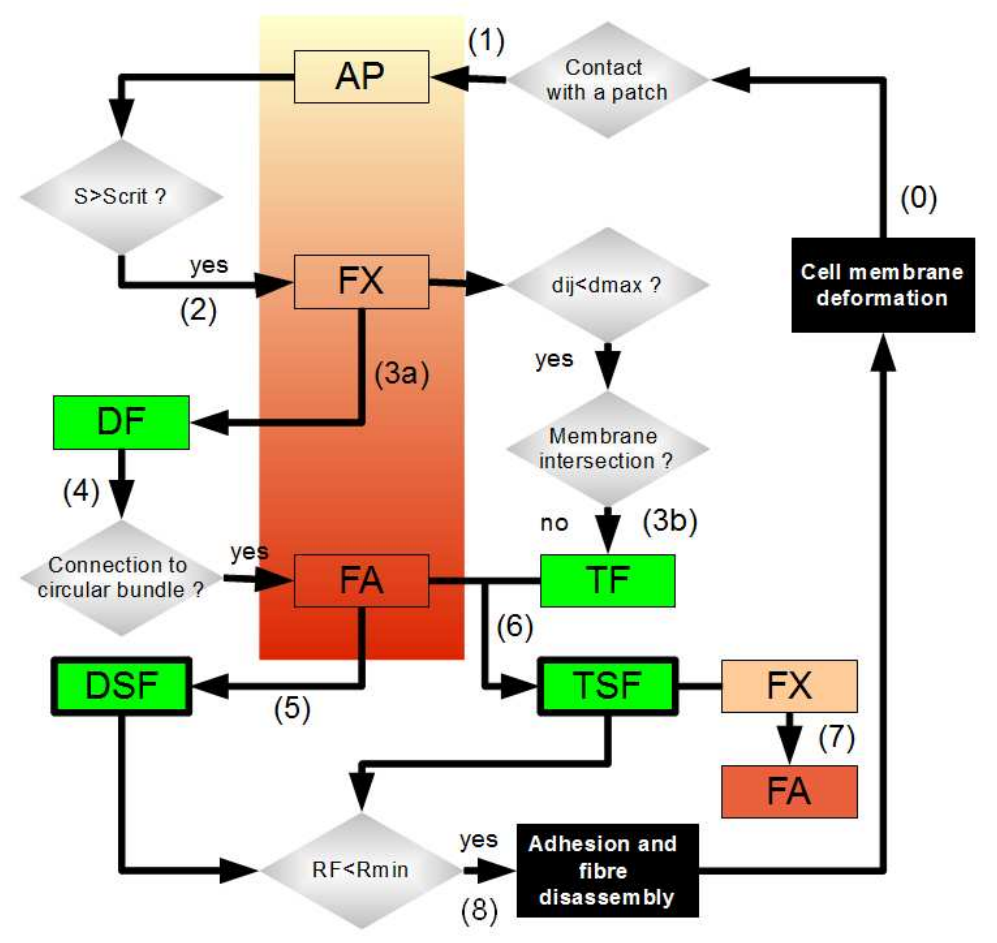

Figure 5: Flowchart describing the formation and maturation of both adhesions and actin cytoskeleton. The membrane bends (0) and APs are recruited as soon as contact with an adhesive patch is established (1). After reaching a critical size $S_{\text {crit }}$, the cluster of APs forms a FX (2). Then two events may occur. In the first case, a DF grows from the FX (3a) until it reaches the circular contractile bundle (4). This contact makes this DF able to exert forces on the FX which matures into FA. This maturation triggers the maturation of the DF into a DSF (5). In the second case, after the formation of an FX (2), if another FX is sufficiently close (closer than $d_{\max }$ ) and if there is space for a filament to form (that is if the membrane is not an obstacle, this is translated in the model by no intersection between the fibre and the membrane), a TF spontaneously grows between the two neighbouring FXs (3b). If a TF is connected to a FA at one end, the TF matures into a TSF (6) and in turn, the FX at the other end of the TSF matures into FA (7). The resulting force $R_{\mathrm{F}}$ exerted by the stress fibres (DSF and TSF) on the adhesions sites is responsible for their becoming (8). If the force is too weak (below $R_{\min }$ ) then the adhesion and related fibres disassemble. 


\subsection{The circular bundle and fibre survival}

The formation of the $\mathrm{CB}$ is not explicitly described in our framework. It is assumed that while the cell starts to spread on the substrate, transverse arcs generated in the lamellipodium thanks to the turnover of the branched actin network, flow from the cell periphery to the cell centre and spontaneously gather to form the $\mathrm{CB}$ according to the scenario proposed in [27]. The CB is therefore quickly appearing during the first few iterations of the simulated cell.

The role of the CB, as demonstrated by Senju and Miyata (2008)[27], is essential for the organisation of the cell stress fibres which will be ultimately responsible for shaping the cell architecture at the stationary state. Based on these experimental observations, we integrate within our computational framework, the key events, which are the maturation of DF into DSF once they connect to the CB. In this scenario the CB, which is a contractile structure, "transmits" its contractile properties to the fibres, which in turn allow the adhesions to mature.

Each time a new fibre connects to the $\mathrm{CB}$, it pulls on it as a spring and induces distortion and rotation. In the model, we choose to describe the bundle as a "deformable" ellipse. At each iteration, the displacement of each bundle point due to the traction of the fibres is calculated. Then the scattered set of displaced points are fitted with an ellipse using a linear least-squares method.

As the CB moves and deforms, some fibres initially linked to it can break whereas other fibres are now close enough to connect to it. In order to decide on the fate of each fibre, a set of criteria is defined as follows:

- we first evaluate the rotation angle of the ellipse. If it is bigger than 45 degrees then we proceed with the next criterion otherwise the connections with the fibres can be broken or created depending on the new distance between the filament ends and the bundle (Fig. 6(1)).

- second, if the DSFs form an angle smaller than 45 degrees with the major axis of the ellipse, then DSFs are maintained otherwise they are disassembled (Fig.6(2)).

\subsection{Spatiotemporal scaling of the model}

Spatial scaling is based on measurements performed on 3T3 fibroblasts. The radius $R_{0}$ of the cell nucleus which remains relatively constant from one cell to another is chosen as the reference length for our model to convert pixels into micrometers. Measurements give $R_{0}=7 \mu \mathrm{m}$.

Temporal scaling is a more delicate issue. We need to identify a process with the appropiate time scale to account for both fast and quick events in the cell. We choose as the reference time scale the elongation rate of the DF, which has been measured experimentally at $0.34 \pm 0.15 \mu \mathrm{m} / \mathrm{min}$ $[22,27]$. With this value, we identify the time with the number of iterations it takes in the simulation to grow such a fibre on the typical length of $R_{0}=7 \mu \mathrm{m}$. According to the growth rate this time corresponds to $21 \mathrm{~min}$ for 600 iterations, which means that 1 iteration corresponds to $0.03 \mathrm{~min}$. 

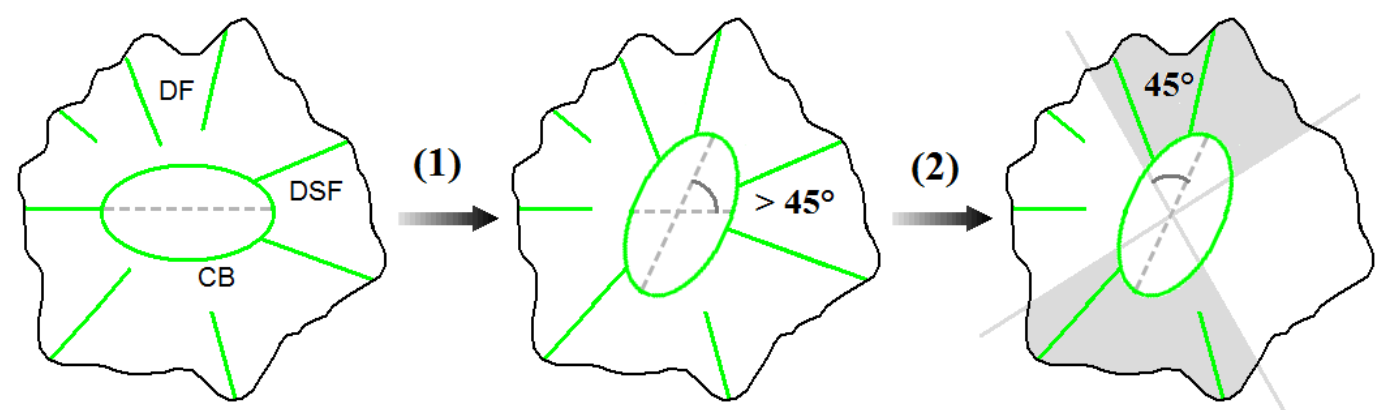

Figure 6: Schematic drawing of the cell to explain the rules managing the assembly or disassembly of DSF. DFs make connections to the circular bundle CB and thus become DSFs which distort it. The new position, shape, and rotation angle of the ellipse major axis are calculated. (1) If the angle of rotation is bigger than \pm 45 degrees, we apply another criterion angle wich governs DSF disassembly. (2) Following such a rotation of the bundle, if the angle formed between the major axis of the bundle and the DSFs is bigger than \pm 45 degrees then the DSFs are disasembled (outside the grey areas).

\section{Results}

The aim of the simulation results presented in this section is two-fold. First, we focus on the importance of the distance between consecutive adhesive patches on the resulting cell morphologies and their stability. Second, we look in more details how the resulting shape is related to the mean force generated by the fibres on the focal adhesions. The influence of the parameter $\psi$ governing the intensity of cell contractility is investigated.

The simulations are performed by changing the parameters $\beta$ and $\gamma$ ruling the balance between membrane protrusion and retraction respectively. This allows to account for a wider range of potentiel cell phenotypes. For example, a protrusive phenotype characterized by extensive cell spreading with longer and more localised membrane extension can be simulated with $\beta>\gamma$ (protrusion dominates over retraction). In the other hand, less protrusive cells, that is with smaller membrane extensions and more rounded shapes are simulated with $\beta=\gamma$. Fibroblasts are typically good representatives of the protrusive phenotype, since these cells spread extensively with large membrane protrusions when plated in vitro on a 2D substrate. In the other hand and in the same experimental 2D in vitro conditions, keratinocyte fits well with the weakly protrusive phenotype. We will therefore use these two representative cell types to illustrate and compare the two protrusive phenotypes in our simulation results.

In our simulations of cell deformation, the initial condition corresponds to a small random perturbation $\epsilon(|\epsilon| \leq 0.05)$ of the homogeneous steady state, with:

$$
L(\theta, 0)=1 \quad a(\theta, 0)=1 \pm \epsilon \quad v(\theta, 0)=0
$$


This corresponds to the state of a cell freshly seeded on the patterned substrate right after trypsinization from its culture dish. The cell shape is circular and actin density relatively homogeneous. At this time, neither adhesions nor actin structures are formed.

The parameters of the model used in the numerical simulations are given in appendix C.

\subsection{Influence of the inter-patches distance on cell morphology}

The deposition of a fibroblast-type cell $(\beta>\gamma)$ on a patterned substrate with an inter-patches distance of $10 \mu \mathrm{m}$ is simulated. The simulation presented in Figure 7 shows the initial round cell which spreads on the patterned substrate over time by forming adhesions and fibres to ultimately reach a constrained steady state in about 71 minutes. Initially, the membrane making contact with the substrate as it spreads, induces the formation of APs at adhesive sites (Fig. 7.A). At this early stage of cell spreading, TAs flow towards the cell centre where they coalesce to form the CB (Fig. 7.B). Although this phenomenon is represented graphically in the simulation, it is not described explicitely in the model. APs mature into FXs as soon as they reach a critical size. Concomitantly to APs maturation into FXs, TFs form between consecutive FXs while DFs emerging from the FXs start to grow radially (Fig. 7.B). Once the DFs reach the CB they allow FXs to mature into FAs and consequently become DSFs. As a result, all TFs related to newly formed FAs mature into TSFs (Fig. 7.C). The maturation of adhesions and fibres leads to a fully constrained polygonal cell shape where the membrane is "trapped" by the underlying TSFs (Fig. 7.D).
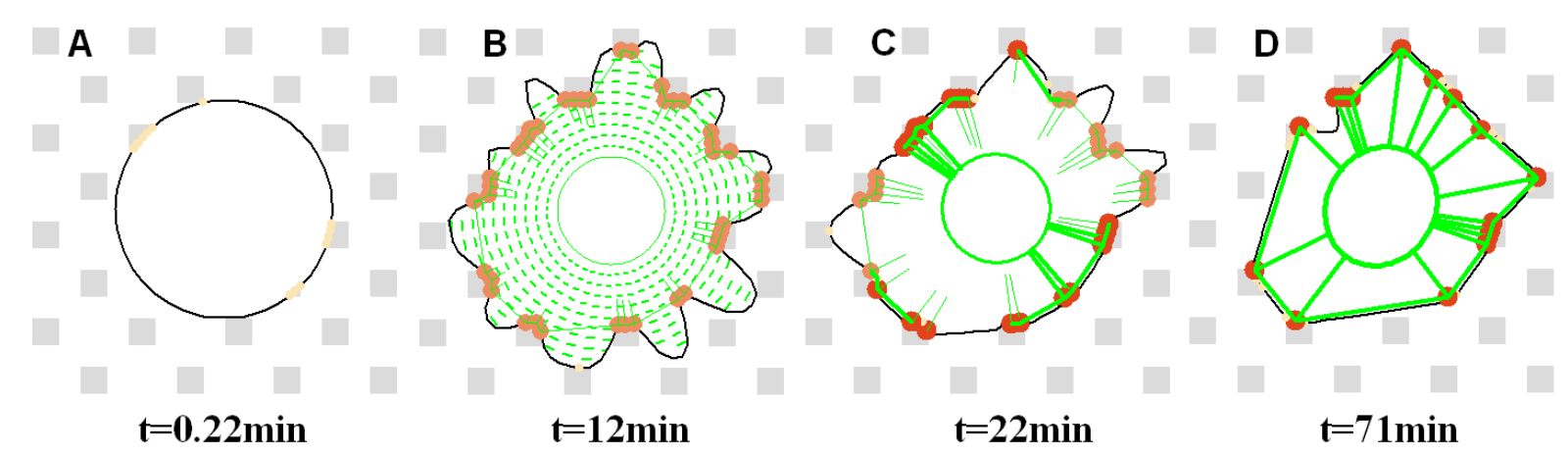

Figure 7: Simulation of the different spreading phases of a cell on an adhesive network with an inter-patches distance $d=10 \mu m(\beta=0.8, \gamma=0.5, \psi=5.4)$. The four main phases are the cell deposition on the subtrate (A), the formation of the $\mathrm{CB}(\mathrm{B})$, the maturation of the adhesions and fibres (C) and the resulting stationary state (D). The different types of adhesions (APs in yellow, FXs in orange and FAs in red) and actin structures (thin lines: non-contractile fibres; thick lines: contractile fibres) are depicted.

On a qualitative basis, the simulated evolution of the cell shape (Fig. 7) strongly resembles our experimental observation of a cell plated on the same patterned substrate (Fig. 3). Although the process starts much quicker in the simulation, since the cell is assumed to make immediate links 
with the substrate. Experimentally, the cell needs first to recover from the trypsinization process.

Figure 8 presents the different resulting cell states obtained depending on the inter-patches distance $d$ of the adhesive pattern from 10 to $24 \mu \mathrm{m}$. For each case, about ten cells have been simulated by virtually seeding each of them at a random location relative to the adhesive pattern. The resulting shapes are different while keeping the same typical features as shown by the two cells represented in each case. The simulated cells generally tend to adopt polygonal shapes dictated by the distribution of the adhesions on the underlying adhesive pattern. As $d$ increases, the cell shapes are less constrained, part of the membrane remains free to move since no transverse fibres could be generated to constrain it. The increased inter-patches distance makes it more difficult for the cell to create FAs. Consequently the cell membrane is only partially constrained, which means that part of the membrane remains free to move (protrude and retract). The cell thus either quickly reaches a fully constrained stable stationary state for small inter-patches distance up to $14 \mu m$ (Fig. 8.A, B, C) or an oscillating state for bigger values of $d$, where the membrane forms pikes that explore the environment in the search of adhesive zones (Fig. 8.D, E, F, G). Transitory adhesions occur, but none are able to mature since the membrane retracts before a fibre had time to form and stabilize the membrane. Figures 8.H and 8.I show the evolution of the cell spreading area with time for two oscillating cells. The curves show that the membrane exploration process is mostly random for these two cells. However more periodical oscillations can also be observed for more constrained cell states.

Figure 9 compares the evolution of the cell spreading area with time for a range of inter-patches distances from $d=10$ to $20 \mu \mathrm{m}$ and for two protrusion/retraction balance rates $\beta=\gamma$ and $\beta>\gamma$ which are used to approach keratinocyte and fibroblast phenotypes respectively.

The mean area of the cells measured at the stationary state is obviously bigger for an increased protrusion/retraction rate. A $60 \%$ increase of this rate leads to a $37 \%$ increase in the mean cell area from $554 \pm 59 \mu m^{2}$ for $\beta=\gamma=0.5$ (Fig. 9.A, C, E, G) to $758 \pm 54 \mu m^{2}$ for $\beta>\gamma$ with $\beta=0.8$ and $\gamma=0.5$ (Fig. 9.B, D, F, H). However despite this difference in the protrusion/retraction rate, used to account for different cell phenotypes, the cells exhibit stable stationary states for inter-patches distances $d$ up to $12 \mu \mathrm{m}$ corresponding to fully constrained cell morphologies (Fig. 9.A, B, C, D). For larger $d$, the cells exhibit oscillating stationary states around a mean surface area (Fig. 9.E, F, G, H). As a consequence, it appears that as long as the cell is able to develop stable adhesions and fibres, it can be fully constrained by its environment whatever its ability to protrude and retract its membrane (that is, its phenotype) provided that the adhesive patches are not too far apart. Indeed, when $d$ is small, the cell can easily find sites to form multiple adhesions in all directions as it spreads. However as $d$ increases, the cell has difficulties to reach multiple adhesion sites in an homogeneous way. Therefore the cell adhesions and fibres mature anisotropically, favouring the direction where the first adhesive sites were encountered. The membrane is thus only partially constrained since the free membrane cannot reach the most distant adhesive sites or if it can, it cannot grow DFs quickly enough, that is before the FXs are disassembled due to their limited lifetime. 

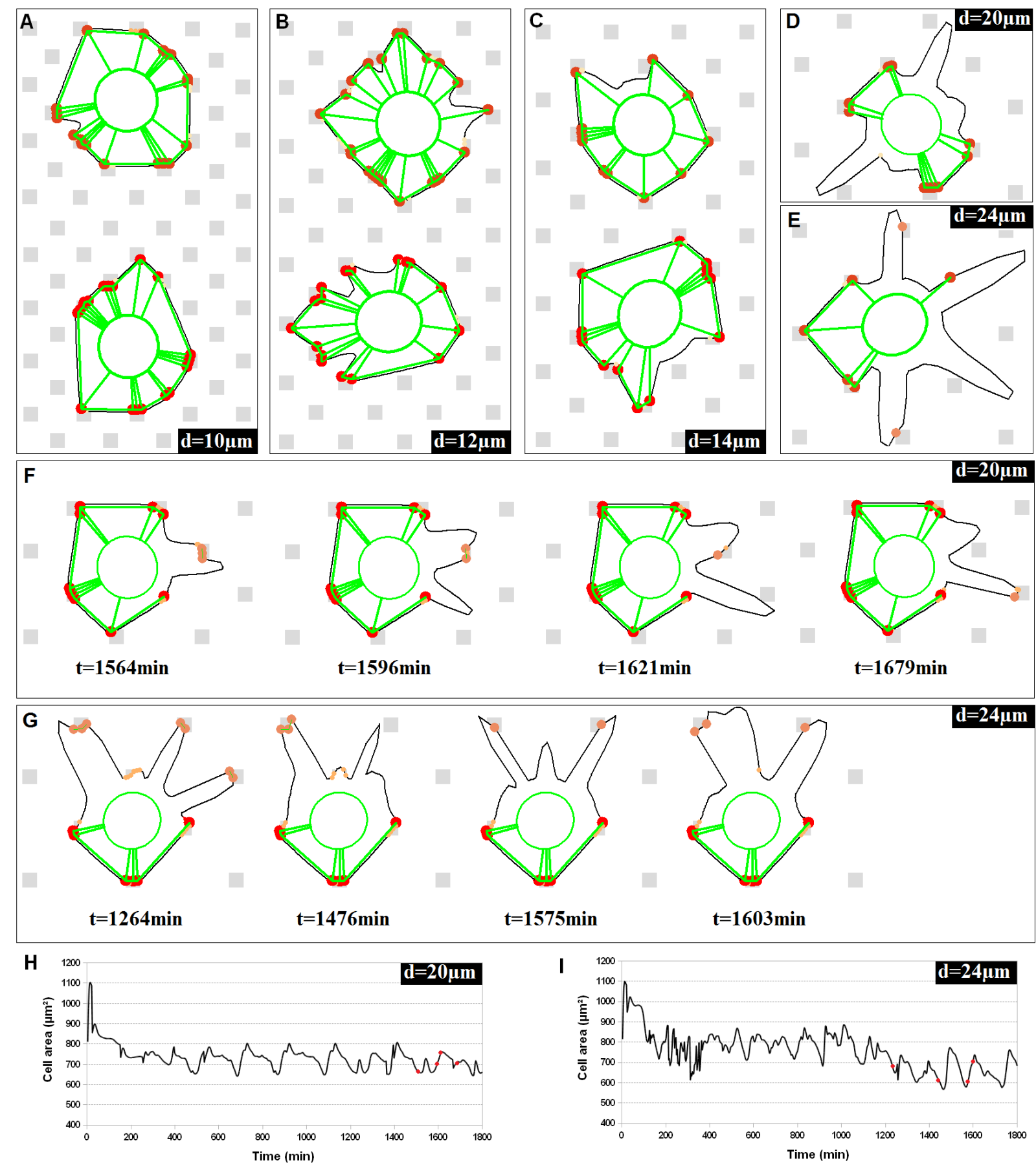

$\mathbf{d}=\mathbf{2 4 \mu \mathrm { m }}$

Figure 8: Resulting cell morphologies simulated for different inter-patches distances $d(\beta=0.8$, $\gamma=0.5, \psi=5.6$ except for A (top), C (bottom) and F where $\psi=5.4$ ). Stationary states are reached at times: $t=63 \mathrm{~min}$ (A, top) $t=59 \mathrm{~min}$ (A, bottom), $t=71 \mathrm{~min}(\mathrm{~B}$, top), $t=126 \mathrm{~min}$ (B, bottom), $t=120 \mathrm{~min}$ (C, top), $t=101 \mathrm{~min}(\mathrm{C}$, bottom), $t=363 \mathrm{~min}(\mathrm{D})$ and $t=294 \mathrm{~min}(\mathrm{E})$. Cells in $\mathrm{D}, \mathrm{E}, \mathrm{F}$ and $\mathrm{G}$ are only partially constrained and the free membrane keeps oscillating. $\mathrm{H}$ and I show the oscillations associated with cells in $\mathrm{F}$ and $\mathrm{G}$ respectively. The spots on the curves $\mathrm{H}$ and I correspond to the snapshots in sequences $\mathrm{F}$ and $\mathrm{G}$. 


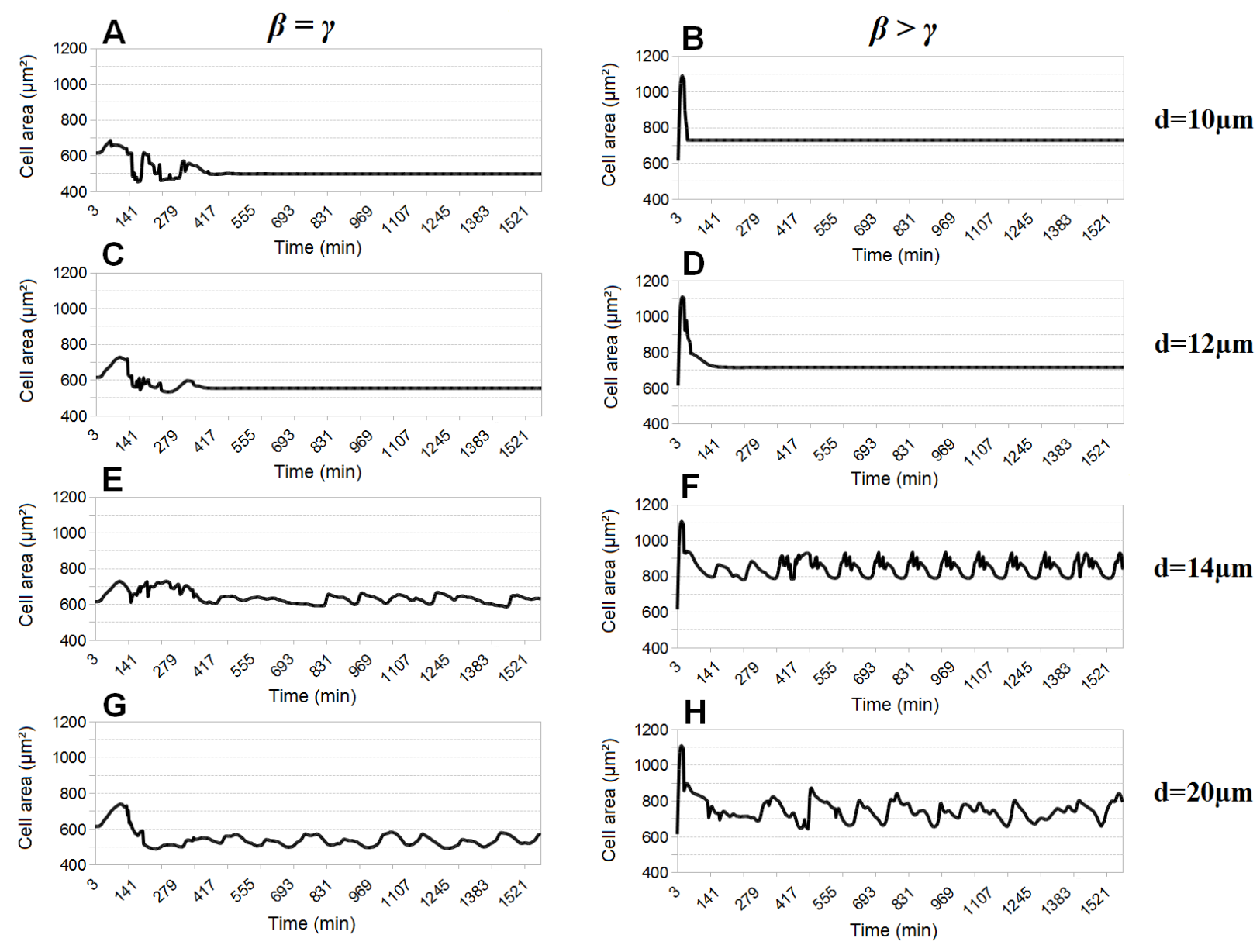

Figure 9: Temporal evolution of the cell area for different inter-patches distances $d$ (with $\psi=5.6$ ). Left column: $\beta=\gamma=0.5$; right column: $\beta>\gamma$ with $\beta=0.8$ and $\gamma=0.5$.

\subsection{Influence of cell contractility on FA maturation and force generation}

FA adhesions survival depends on a mechanical force. If the resulting traction force exerted on a FA is below a threshold force $\left(R_{\mathrm{F}}<R_{\text {min }}\right)$, then the adhesion disassembles. Thus to investigate the forces at stakes in the cell, it is first necessary to assess the conditions that lead to the maturation of the cell adhesions into FAs.

Figure 10 shows that for small inter-patches distance up to $d=12 \mu \mathrm{m}$ more than $80 \%$ of the FXs formed mature into FAs, whatever the protrusion/retraction balance (i.e. cell phenotype) and cell contractility. For the protrusive phenotype (i.e. fibroblast-like) the maturation rate is even higher, above $90 \%$ (Fig. 10.B).

For higher values of $d$, more heterogenous cases are found with some cells maintaining a high 
level of maturation of their FXs into FAs and some other with a significantly lower maturation rate down to $60 \%$ for $d=14 \mu \mathrm{m}$. This goes further down as $d$ increases to $20 \mu \mathrm{m}$ with $40 \%$ for $\beta=\gamma$ and $20 \%$ for $\beta>\gamma$, uncorrelated with the contractility $\psi$.

It appears that FXs maturation into FAs is high for constrained cell shapes whereas stationary oscillating states are rather correlated with smaller maturation rates. The discrepancies for the FAs maturation rate observed for higher inter-patches distance can be explained as follows:

- if the free cell membrane while oscillating, encounters an adhesive patch then it can form FXs. However these FXs cannot mature into FAs if the time it takes for the DFs to grow exceed the adhesion lifetime. That happens if the adhesion is located too far away from the cell body. In that case cell membrane oscillations lead to a sharp decrease of the FAs maturation rate, since lots of FXs are formed in the course of membrane oscillations but unsuccessful to mature into FAs. This is mainly the case for the protrusive phenotype where the cell membrane explores a wider environment(Fig. 10.B).

- on the other hand, if the free cell membrane oscillates without meeting an adhesive patch, then the maturation rate of FXs into FAs is high since it only corresponds to the quick and successful maturation process related to the constrained side of the cells. It is the case for non-protrusive phenotype where the movements of the membrane are reduced and the cell cannot make adhesions with distant patches. Consequently less adhesions are formed but all of them mature into FA, either quickly for higher contractility through contractile fibre "contamination" or slowly for lower contractility thanks to the reduced movements of the membrane that preserve the forming adhesions. Intermediate contractility can potentially leads to intermediate situations with decreased maturation (Fig. 10.A).

These simulation results tend to suggest that the contractility plays a secondary "modulating" part in the adhesion maturation process. The interpatch distance and the phenotype are more important according to the model.

Figure 11 presents the evolution of the mean traction force exerted by the stress fibres (DSF and TSF) on the FAs as a function of the inter-patches distance $d$. The curves represent the average values of measurements performed in three simulated cells. Once again, two different balances for the protrusion/retraction (i.e. cell phenotypes) are considered as well as three different values for the cell contractility coefficient $\psi$.

The forces generated by the stress fibres on the FAs are relatively homogeneous since they do not vary by more than $10 \%$ around a mean value of $2.15 n$.u.

Numerical simulations reveal that, whatever the protrusion/retraction balance and cell contractility, the traction force generally tends first to increase with $d$, reach a plateau and then decrease as $d$ keeps on increasing. Considering that the forces are generated by the DSFs and TSFs, both modelled as springs whose tension magnitude is proportional to their length, the bell-shape profiles can be explained as follows: 

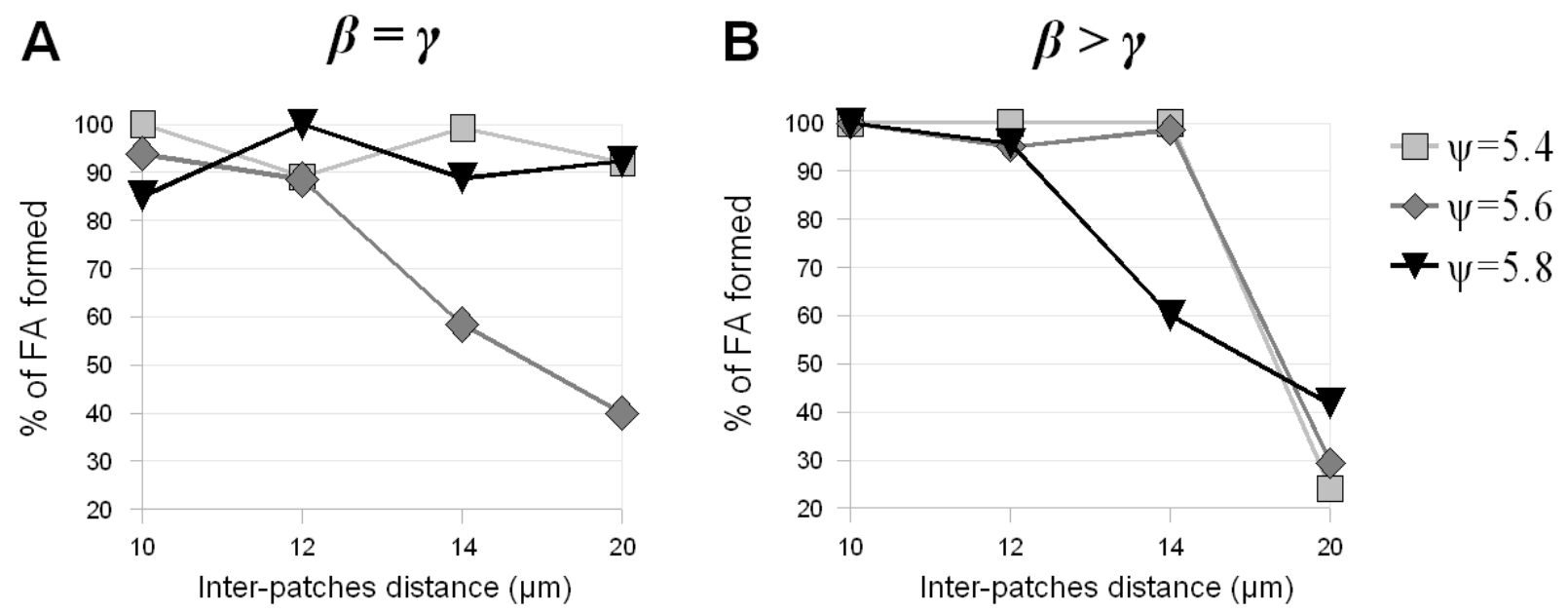

Figure 10: Percentage of FXs which have maturated into FAs, evaluated at the stationary state, as a function of the inter-patches distance $d$. (A) $\beta=\gamma=0.5$; (B) $\beta>\gamma$ with $\beta=0.8$ and $\gamma=0.5$. Three values of the cell contractility coefficient $\psi$ are considered in each case.

- if $d$ is small $(d<12 \mu m)$, the lengths of the DSFs and TSFs are also small and so are the generated tension forces,

- if $d$ is intermediate ( $12 \leq d \leq 14 \mu m$ ), the lengths of the stress fibres increase which induces a higher magnitude of forces,

- finally, if $d$ is big ( $d>14 \mu m)$, as seen previously, it becomes more difficult for the cell to establish homogeneously stable adhesions and fibres. Consequently, the cell shape is more anisotropic with a partially constrained area and a partially free membrane. The constrained area is developing next to the CB, which means that many more shorter DSFs are created and very few longer TSFs (Fig. 8.D, E). This results in the decrease of the magnitude for the mean traction force.

Whereas the curves profiles for different values of the contractility $\psi$ remain very close in evolution and magnitude, for the protrusive phenotype (i.e. fibroblast-like), they differ quite significantly in magnitude for the other keratinocyte-like phenotype. In that latter case, the maximum of the mean traction forces (corresponding to $d=12 \mu \mathrm{m}$ ) increases with the cell contractility from 2.10 up to $2.36 n$.u., which corresponds to a $0.26 n$.u. increase between $\psi$-curves. For the fibroblast case, the maximum force difference is twice smaller with $0.13 n . u$.

This phenomenon is mostly explained by the fact that the contractility effect is overcome by the predominantly protrusive cell phenotype. Large membrane extensions dominate the cell movements and the contractility only plays a reduced role in the force generation according to the model. 

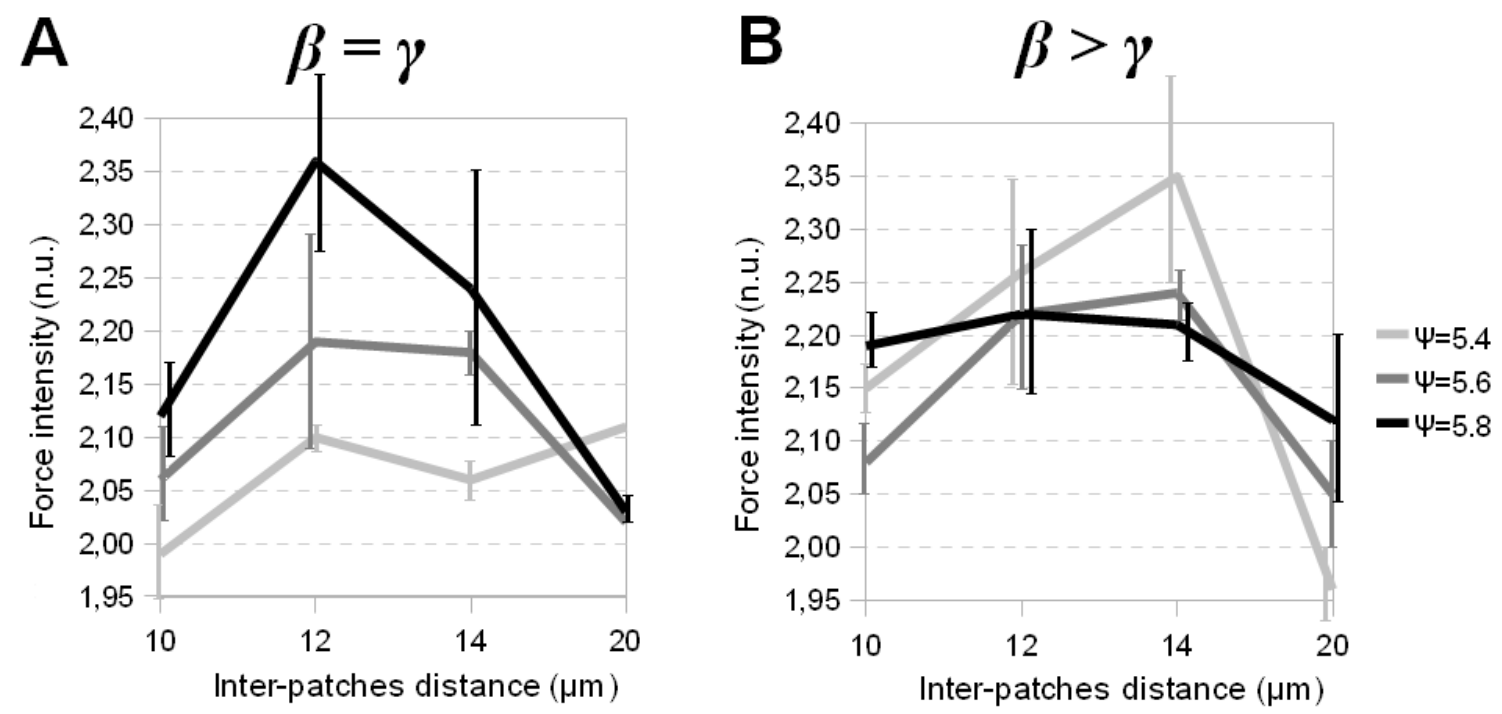

Figure 11: Evolution of the mean traction force (in normalized units: n.u.) from the stress fibres on each FA for different inter-patches distance $d$ and evaluated at the cell stationary state. Measurements are averaged for 3 simulated cells for each point of the curves (vertical bars represent the standard deviations around the mean values). (A) $\beta=\gamma=0.5$; (B) $\beta>\gamma$ with $\beta=0.8$ and $\gamma=0.5$. Three values of the cell contractility coefficient $\psi$ are considered in each case.

\section{Conclusions}

In this paper, we propose a model that couples the formation, maturation and turnover of cell adhesions and actin fibres. For that we take into account the successive stages of adhesion maturation from adhesion precursors to focal complexes and ultimately to focal adhesions; as well as the actin fibres evolution from the growing filaments, their bundling and the acquisition of contractility to form the stress fibres. The two events are closely interdependent and coordinated as recently highlighted by a number of studies $[27,22]$ which serve as a basis for the development of our computational model.

Since our aim is to investigate the relationship between adhesions and fibres, a means to control the position of the former and the resulting architecture of the latter is to use specifically engineered substrates to discretize the cell adhesive environment. For that a network of regularly arranged adhesive patches is considered. This allows to isolate and position the different adhesive sites in the cell from which the fibres grow and consequently allows to generate more organized fibre structures. It is therefore easier to decipher the mechanisms ruling the cell shape through the control acquired on them to generate this shape. Moreover, the use of such patterned substrate gives us a mean to investigate the impact of the environment on the cell internal organization, by varying the distance between consecutive adhesive patches.

In a first stage, simulations were realised to calibrate the model parameters in order to qualita- 
tively match our experimental observations of cell spreading on adhesive patches. The model was successful to reproduce the successive spreading phases and the resulting constrained cell shape, where the square shape obtained for the virtual cell resembles the real cell for the same environmental conditions.

We then proposed to enlarge our analysis with the systematic comparison between two different cell phenotypes. Those could be approached by tuning the model parameters defining the cell intrinsic mechanical properties. In that specific case, acting on the parameters which govern the protrusion/retraction balance on the membrane, allows to differentiate two major cell types, a protrusive type and a non-protrusive one. The protrusive type characterizes fibroblast cells, which easily spread on 2D substrates with large membrane extensions. The non-protrusive type on the other hand, fits well with keratinocytes which are round-shape cells, with narrow membrane extension on $2 \mathrm{D}$ substrates.

The simulations realised revealed that whatever the cell phenotype (which more precisely reflects here the cell protrusive potential), the cell shape is essentially governed by the inter-patches distance of the adhesive pattern. For distances up to $12 \mu \mathrm{m}$ the simulated cell shapes are fully constrained by the stress fibres and the cell morphologies are mostly geometrical and stable. However for higher inter-patches distances the cell is unable to homogeneously develop stress fibres and the resulting shapes are only partially constrained. Free partial cell membrane movement leads to cell shape oscillations.

One crucial point of this work is the question of force generation in the cell. For that we evaluated the mean traction force generated at each focal adhesions. We investigated in particular the influence of the parameter governing the magnitude of the cell contractility. We found that the level of force remains relatively homogeneous, with only a $10 \%$ maximum variation around the mean value. However the force versus inter-patches distance profiles, interestingly revealed bell-shaped curves, increasing for small distances and decreasing for large ones with a maximum always found for the critical distance of $12 \mu \mathrm{m}$. Indeed the size of the stress fibres which generate the tension forces depends on the inter-patches distance. Small distance leads to small fibres with reduced forces whereas larger distances lead to longer fibres and higher forces up to the point where the fibres cannot form homogeneously anymore in the cell. The lifetime of the adhesion supporting their growth being limited, long fibres cannot mature quickly enough, thus limiting the formation of the stress fibres on distant patches and the capacity for the cell to develop higher forces. We moreover observed that the cell contractility modulates the level of force generated by the cell only for the non-protrusive keratinocyte phenotype. This modulation role is not visible for the protrusive fibroblast phenotype, since contractility is most likely dominated by the protrusive effect in that case.

We show by varying the distance between the adhesive patches of the patterned substrate, that the adhesion lifetime and the growing rate of the fibres are the key parameters conditioning the cell morphologies and their stability (either constrained or oscillating). Guillou et al. (2008) [13] al- 
ready suggested that a critical and limited filament length exists in the cell. We further suggest that this length could also depend on the combination between the adhesion lifetime and the filament growing rate. Future experimental work to specifically investigate this aspect is awaited. On the theoretical side, the model also deserves further refinements. Fibrillar adhesions for example have not been considered since this adhesion type is mainly located out of the cell periphery $[3,7,40]$. Additionally, more cytoskeletal components than actin structures should be incorporated. Indeed, microtubules have been shown to play a key role in the coordination of adhesion disassembly $[3,38]$.

\section{Acknowledgements}

We would like to thank D. Fuard for technical assistance making the masks for adhesive patch printing. We are specially grateful to Dr E. Mylona for helpful comments concerning the biological aspects of this paper and critical reading of the manuscript.

\section{Appendix}

\section{A Cell culture and videomicroscopy}

Swiss mouse 3T3 fibroblasts were cultured in Dulbecco's Modified Eagles Medium (DMEM, Sigma Aldrich) supplemented with $10 \%$ foetal calf serum (Invitrogen), $1 \%$ L-glutamine and $0.25 \%$ penicillin/streptomycin. Cells were seeded on the patterned substrate and allowed to adhere for one hour. They were then placed on the miscroscope stage for image acquisition. An inverted Axiovert 135 microscope (Carl Zeiss, Jena, Germany) equipped with an incubating chamber was used to maintain the cells in physiological conditions at $37^{\circ} \mathrm{C}$ and $5 \% \mathrm{CO}_{2}$ in a wet atmosphere. Images were recorded every 5 minutes with a CDD coolsnap camera (Roper Scientific) for 8 hours.

\section{B Microfabrication of the Adhesive Patches}

Cleaned glass slides are rendered hyper hydrophilic (water contact angle of $20^{\circ}$ ) through successive ethanol washing and oxygen plasma treatment. Then, slides were uniformly coated with a $1.5 \mu \mathrm{m}$ thick UV-sensitive film and irradiated at specific places to form patches of hydrophilic zones through a chromium mask. The patches were rendered highly hydrophobic by vapour deposition of a commercial fluoro silane product, OPTOOL DSX ${ }^{\mathrm{TM}}$ from Daikin. After silane treatment the polymerized resist was dissolved with acetone. The adhesive patches do not require to be functionalized with matrix proteins for the cell to adhere. We therefore used such uncoated (extracellular matrix free) substrates in our experiments.

Figure 12 shows a picture of the patterned substrate we used, before removal of the resist (patches become invisible after removal). 


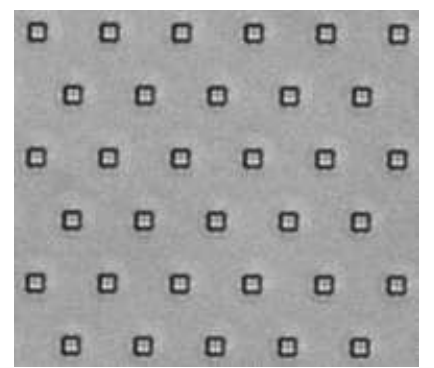

Figure 12: Network of adhesive patches with an inter-patches distance of $12 \mu \mathrm{m}$ and a patch size of $4 \times 4 \mu m^{2}$.

\section{Simulation parameters}

The parameters appearing in the system of partial differential equations (eqs. 3.3) and defining the cell mechanical properties including viscoelasticity $(\mu, \gamma)$, contractility $(\psi)$, friction $(\alpha)$ and protrusion $(\beta)$ which govern the cell movements are dimensionless parameters (Table 1). Details on the normalization of the system and choice of these parameter values and dimension can be found in [31, 32]. The coefficients used to describe the tension forces of the membrane and fibres have been adjusted relatively to the other mechanical parameters to correspond to a realistic range of values. For example, the elasticity of the fibres $k_{f}$ is chosen in the same range as the elasticity of the actin network $\gamma$. The elasticity of the membrane being much smaller than the one of the fibres, the value $k_{m}$ is chosen accordingly.

\begin{tabular}{lcc}
\hline Parameter & notation & value \\
\hline actin network elasticity & $\gamma$ & 0.5 \\
cytoplasm viscosity & $\mu$ & 2.0 \\
protrusive hydrostatic pressure & $\beta$ & 0.5 or 0.8 \\
cell contractility & $\psi$ & 5.4 to 5.8 \\
saturation actin density & $a_{\text {sat }}$ & 2.0 \\
APs friction coefficient & $\alpha$ & 0.05 \\
repulsion coefficient from the CB & $\beta_{b}$ & $2 \beta$ \\
membrane elasticity & $k_{m}$ & 0.05 \\
actin fibre elasticity & $k_{f}$ & 0.2 \\
maximum resulting traction force & $R_{\min }$ & 0.6 \\
\hline
\end{tabular}

Table 1: Dimensionless parameters defining the cell biomechanical properties used in the numerical simulations.

The length parameters have been evaluated from experimental observations (Table 2). The critical size of the AP correspond to $300 \mathrm{~nm}$. We consider that a cluster is formed from four APs to estimate the critical size $S_{\text {crit }}$ from which a FX is formed. Finally, one crucial parameter is the 
lifetime of the FX. It is admitted that it is about a few minutes [39], whereas the more stable FA can be sustained from 30 to 90 minutes [2]. In the model, since we do not describe the molecular cascade for adhesion regulation, we do not limit the lifetime of the FA however, we set the lifetime for FX to the lower boundary of 30 minutes.

\begin{tabular}{lccc}
\hline Parameter & notation & value & units \\
\hline radius of the cell nucleus & $R_{0}$ & 7 & $\mu \mathrm{m}$ \\
membrane segment resting length & $l_{0}$ & 0.9 & $\mu \mathrm{m}$ \\
fibre resting length & $l_{0 f}$ & 7 & $\mu \mathrm{m}$ \\
maximum distance allowed between FXs & $d_{\max }$ & 22 & $\mu \mathrm{m}$ \\
critical size of the APs cluster & $S_{\text {crit }}$ & 1.2 & $\mu \mathrm{m}$ \\
FX lifetime & $\tau_{F X}$ & 30 & $\mathrm{~min}$ \\
\hline
\end{tabular}

Table 2: Spatiotemporal parameters defining the characteristics lengths and time used in the model simulations.

\section{References}

[1] I. Bischofs, F. Klein, D. Lehnert, M. Bastmeyer, U. Schwarz. Filamentous network mechanics and active contractility determine cell and tissue shape. Biophys. J, 95 (2008), 3488-3496.

[2] M. Block, C. Badowski, A. Millon-Fremillon, D. Bouvard, A. Bouin, E. Faurobert, D. Gerber-Scokaert, E. Planus, C. Albigès-Rizo. Podosome type adhesions and focal adhesions, so alike and yet so different. Eur. J. Cell Biol., 87 (2008), 491-506.

[3] J. Broussard, D. Webb, I. Kaverina. Asymmetric focal adhesion disassembly in motile cells. Curr. Opin. Cell Biol., 20 (2008), 85-90.

[4] H. Coskun, Y. Li, M. Mackey. Ameboid cell motility: A model and inverse problem, with an application to live cell imaging data. J. Theor. Biol., 244 (2007), 169-179.

[5] V. Deshpande, R. McMeeking, A. Evans. A bio-chemo-mechanical model for cell contractility. PNAS, 103 (2006), 14015-14020.

[6] V. Deshpande, R. McMeeking, A. Evans. A model for the contractility of the cytoskeleton including the effects of stress-fibre formation and dissociation. Proc. R. Soc. A, 463 (2007), 787-815.

[7] A. Efimov, N. Schiefermeier, I. Grigoriev, M. Brown, C. Turner, J. Small, I. Kaverina. Paxillin-dependent stimulation of microtubule catastrophes at focal adhesion sites. J. Cell Sci., 121 (2008), 196-204. 
[8] A. Engler, S. Sen, H. Sweeney, D. Discher. Matrix Elasticity Directs Stem Cell Lineage Specification. Cell, 126 (2006), 677-689.

[9] P. Friedl, K. Wolf. Tumour-cell invasion and migration: diversity and escape mechanisms. Nat. Rev. Cancer, 3 (2003), 362-374.

[10] C. Galbraith, K. Yamada, M. Sheetz. The relationship between force and focal complex development. J. Cell Biol., 159 (2002), No. 4, 695-705.

[11] B. Geiger, J. Spatz, A. Bershadsky. Environmental sensing through focal adhesions. Nat. Rev. Mol. Cell Biol., 10 (2009), 21-33.

[12] G. Giannone, B. Dubin-Thaler, O. Rossier, Y. Cai, O. Chaga, G. Jiang, W. Beaver, H. Dobereiner, Y. Freund, G. Borisy, M. Sheetz. Lamellipodial actin mechanically links myosin activity with adhesion-site formation. Cell, 128 (2007), 561-575.

[13] H. Guillou, A. Depraz-Depland, E. Planus, B. Vianay, J. Chaussy, A. Grichine, C. AlbigèsRizo, M. Block. Lamellipodia nucleation by filopodia depends on integrin occupancy and downstream Racl signaling. Exp. Cell Res., 314 (2008), 478-488.

[14] P. Hotulainen, P. Lappalainen. Stress fibers are generated by two distinct actin assembly mechanisms in motile cells. J. Cell Biol., 173 (2006), 383-394.

[15] J. James, E. Goluch, H. Hu, C. Liu, M. Mrksich. Subcellular Curvature at the Perimeter of Micropatterned Cells Influences Lamellipodial Distribution and Cell Polarity. Cell Motil. Cytoskeleton, 65 (2008), 841-852.

[16] G. Jiang, A. Huang, Y. Cai, M. Tanase, M. Sheetz. Rigidity sensing at the leading edge through $\alpha_{v} \beta_{3}$ integrins and RPTP $\alpha$. Biophys. J., 90 (2006), 1804-1809.

[17] R. Kaunas, H. Hsu. A kinematic model of stretch-induced stress fiber turnover and reorientation, J. Theor. Biol., 257 (2009), 320-330.

[18] E. Kuusela, W. Alt. Continuum model of cell adhesion and migration. J. Math. Biol., 58 (2009), 135-161.

[19] K. Lazopoulos, D. Stamenovic. A mathematical model of cell reorientation in response to substrate stretching. Mol. Cell. Biomech., 3 (2006), 43.

[20] J. Lock, B. Wehrle-Haller, S. Strömblad. Cell-matrix adhesion complexes: master control machinery of cell migration. International Journal of Solids and Structures, 18 (2008), 6576.

[21] Y. Luo, X. Xu, T. Lele, S. Kumar, D. Ingber. A multi-modular tensegrity model of an actin stress fiber. J. Biomech., 41 (2008), 2379-2387. 
[22] P. Naumanen, P. Lappalainen, P. Hotulainen. Mechanisms of actin stress fibre assembly. J. Microsc., 231 (2008), 446-454.

[23] A. Pathak, V. Deshpande, R. McMeeking, A. Evans. The simulation of stress fibre and focal adhesion development in cells on patterned substrates. J. R. Soc. Interface, 5 (2008), 507524.

[24] S. Pellegrin, H. Mellor. Actin stress fibres. J. Cell Sci., 120 (2007), 3491-3499.

[25] R. Rid, N. Schiefermeier, I. Grigoriev, J. Small, I. Kaverina. The Last but not the Least: The Origin and Significance of Trailing Adhesions in Fibroblastic Cells. Cell Motil. Cytoskeleton, 61 (2005), 161-171.

[26] A. Saez, M. Ghibaudo, A. Buguin, P. Silberzan, B. Ladoux. Rigidity-driven growth and migration of epithelial cells on microstructured anisotropic substrates. PNAS, 104 (2007), 8281-8286.

[27] Y. Senju, H. Miyata. The role of actomyosin contractility in the formation and dynamics of actin bundles during fibroblasts spreading. J. Biochem., 145 (2008), 137-150.

[28] J. Small, S. Auinger, M. Nemethova, S. Koestler, K. Goldie, A. Hoenger, G. Resch. Unravelling the structure of the lamellipodium. J. Microsc., 231 (2008), 479-485.

[29] D. Stamenovic. Contractile torque as a steering mechanism for orientation of adherent cells. Mol. Cell. Biomech., 2 (2005), 69.

[30] A. Stéphanou. A computational framework integrating cytoskeletal and adhesion dynamics for modelling cell motility. Cell Mechanics, From Single Scale-Based Models to Multiscale Modeling. Chapman \& Hall / CRC Press, Ed. A. Chauvire, L.Preziosi \& C. Verdier, 2009.

[31] A. Stéphanou, M. Chaplain, P. Tracqui. A mathematical model for the dynamics of large membrane deformations of isolated fibroblasts. Bull. Math. Biol., 66 (2004), 1119-1154.

[32] A. Stéphanou, E. Mylona, M. Chaplain, P. Tracqui. A computational model of cell migration coupling the growth of focal adhesions with oscillatory cell protrusions. J. Theor. Biol., 253 (2008), 701-716.

[33] J. Tan, J. Tien, D. Pirone, D. Gray, K. Bhadriraju, C. Chen. Cells lying on a bed of microneedles: an approach to isolate mechanical force. PNAS, 100 (2003), 1484-1489.

[34] M. Théry, A. Pépin, E. Dressaire, Y. Chen, M. Bornens. Cell Distribution of Stress Fibres in Response to the Geometry of the Adhesive Environment. Cell Motil. Cytoskeleton, 63 (2006), 341-355.

[35] T. Tzvetkova-Chevolleau, A. Stéphanou, D. Fuard, J. Ohayon, P. Schiavone, P. Tracqui. The motility of normal and cancer cells in response to the combined influence of the substrate rigidity and anisotropic microstructure. Biomaterials, 29 (2008), 1541-1551. 
[36] M. Vicente-Manzanares, C. Choi, A. Horwitz. Integrins in cell migration-the actin connection. J. Cell Sci., 122 (2009), 199-206.

[37] H. Wolfenson, Y. Henis, B. Geiger, A. Bershadsky. The heel and toe of the cell's foot: a multifaceted approach for understanding the structure and dynamics of focal adhesions. Cell Motil. Cytoskeleton, 66 (2009), 1017-1029.

[38] D. Worth, M. Parsons. Adhesion dynamics: Mechanisms and measurements. Int. J. Biochem. Cell Biol., 40 (2008), 2397-2409.

[39] R. Zaidel-Bar, C. Ballestrem, Z. Kam, B. Geiger. Early molecular events in the assembly of matrix adhesions at the leading edge of migrating cells. J. Cell Sci., 116 (2003), 4605-4613.

[40] R. Zaidel-Bar, M. Cohen, L. Addadi, B. Geiger. Hierarchical assembly of cell-matrix adhesion complexes. Biochem. Soc. Trans., 32 (2004), 416-420.

[41] R. Zaidel-Bar, S. Itzkovitz, A. Ma'ayan, R. Iyengar, B. Geiger. Functional atlas of the integrin adhesome. Nat. Cell Biol., 9 (2007), 858-867. 